\title{
Weak charge of the proton and new physics
}

\author{
Jens Erler, ${ }^{1,2, *}$ Andriy Kurylov, ${ }^{3, \dagger}$ and Michael J. Ramsey-Musolf $2,3,4, \$$ \\ ${ }^{1}$ Instituto de Física, Universidad Nacional Autónoma de México, 04510 México D.F., Mexico \\ ${ }^{2}$ Institute for Nuclear Theory, University of Washington, Seattle, Washington 98195, USA \\ ${ }^{3}$ Kellogg Radiation Laboratory, California Institute of Technology, Pasadena, California 91125, USA \\ ${ }^{4}$ Department of Physics, University of Connecticut, Storrs, Connecticut 06269, USA
}

(Received 27 February 2003; published 17 July 2003)

\begin{abstract}
We address the physics implications of a precise determination of the weak charge of the proton, $Q_{W}(p)$, from a parity violating elastic electron proton scattering experiment to be performed at the Jefferson Laboratory. We present the standard model (SM) expression for $Q_{W}(p)$ including one-loop radiative corrections, and discuss in detail the theoretical uncertainties and missing higher order QCD corrections. Owing to a fortuitous cancellation, the value of $Q_{W}(p)$ is suppressed in the SM, making it a unique place to look for physics beyond the SM. Examples include extra neutral gauge bosons, supersymmetry, and leptoquarks. We argue that a $Q_{W}(p)$ measurement will provide an important complement to both high energy collider experiments and other low energy electroweak measurements. The anticipated experimental precision requires the knowledge of the $O\left(\alpha_{s}\right)$ corrections to the pure electroweak box contributions. We compute these contributions for $Q_{W}(p)$, as well as for the weak charges of heavy elements as determined from atomic parity violation.
\end{abstract}

DOI: $10.1103 /$ PhysRevD.68.016006

PACS number(s): 11.30.Er, 25.30.Bf, 12.15.Lk

\section{INTRODUCTION}

Precision tests continue to play an essential role in elucidating the structure of the electroweak (EW) interaction $[1-4]$. Such tests include the complete high energy program on top of the $Z$ resonance at the $e^{+} e^{-}$accelerators, the CERN $e^{+} e^{-}$collider LEP 1 and SLAC Linear Collider (SLC); precision measurements at LEP 2 and the Fermilab $p \bar{p}$ collider Tevatron; and deep inelastic scattering (DIS) at the DESY e $p$ collider HERA [5]. Recent precision measurements at lower energies, such as a determination of the muon anomalous magnetic moment $a_{\mu}[6]$ and of cross sections for neutrino-nucleus DIS [7], have shown deviations from the standard model (SM) expectations and generated some excitement about possible signatures of new physics, although theoretical uncertainties from the strong interaction presently cloud the interpretation of the results [8-13].

In this paper we focus on the prospective impact of a precision low energy measurement of the weak charge of the proton, $Q_{W}(p)$, using parity violating (PV) elastic ep scattering. Such an experiment has recently been proposed [14] and approved at the Thomas Jefferson National Accelerator Facility (JLab) using the Continuous Electron Beam Accelerator Facility (CEBAF). Historically, semileptonic neutral current experiments have contributed substantially to our understanding of the EW interaction. In particular, the deep inelastic $e D$ asymmetry measurement at SLAC [15] in the late 1970s played a crucial role in singling out the SM over its alternatives at that time, and provided first measurements of the effective PV electron-quark couplings, $2 C_{1 u}-C_{1 d}$ and $2 C_{2 u}-C_{2 d}$ (defined in Sec. IV). Subsequently, the latter

\footnotetext{
*Email address: erler@fisica.unam.mx

${ }^{\dagger}$ Email address: kurilov@krl.caltech.edu

‡Email address: mjrm@krl.caltech.edu
}

combination was determined more precisely in DIS of muons from carbon at CERN [16]. Quasielastic and elastic electron scattering, respectively, from ${ }^{9} \mathrm{Be}$ at Mainz [17] and ${ }^{12} \mathrm{C}$ at MIT-Bates [18], constrained the remaining linear combinations. More recently, measurements of the elastic $e p$ and $e D$ asymmetries at MIT-Bates [19] and JLab [20] have been used to derive information on the neutral weak magnetic, electric, and axial vector form factors of the proton at $q^{2} \neq 0$, and yielded a value for $C_{2 u}-C_{2 d}$ [19]. Experiments probing atomic PV (APV) provided further precise information on various linear combinations of the $C_{1 i}$ [21-23]. On the other hand, the neutral weak charge of the proton, proportional to $2 C_{1 u}+C_{1 d}$, has never been measured.

In its own right, $Q_{W}(p)$ is a fundamental property of the proton, being the neutral current analog of the vector coupling $G_{V}$, which enters neutron and nuclear $\beta$ decay. While measurements of $G_{V}$ provide the most precise determination of the Cabibbo-Kobayashi-Maskawa (CKM) matrix element $V_{u d}$, a precise determination of $Q_{W}(p)$ may provide insight into the SM and its possible extensions. Because the value of the weak mixing angle $\sin ^{2} \theta_{W}$ is numerically close to $1 / 4$,

$$
Q_{W}(p)=1-4 \sin ^{2} \theta_{W}
$$

is suppressed in the SM (see Sec. III). This suppression is characteristic for protons (and electrons) but not neutrons, and therefore it is absent in any other nucleus. As a consequence, $Q_{W}(p)$ is unusually sensitive to $\sin ^{2} \theta_{W}$ and offers a unique place to extract it at low momentum transfer. Doing so will provide a test for the renormalization group evolution (RGE) of $\sin ^{2} \theta_{W}$.

To put this statement in context, we note that the strong coupling $\alpha_{s}$ is routinely subjected to analogous RGE tests, whose results provide crucial evidence that QCD is the correct theory of strong interaction. As we discuss in Sec. III, a precise measurement of $Q_{W}(p)$-along with the analogous 
measurement of the weak charge of the electron, $Q_{W}(e)$, currently measured by the E-158 Collaboration at SLAC [24] — will provide this important test for the EW sector of the theory. An observed deviation of the running of $\sin ^{2} \theta_{W}$ from the SM prediction could signal the presence of new physics, whereas agreement would place new constraints on possible SM extensions. This test has taken on added interest recently in light of the $\nu$-nucleus DIS results obtained by the NuTeV Collaboration [7] which show a $3 \sigma$ deviation from the SM prediction. In contrast, the most recent determination of the weak charge of cesium, $Q_{W}(\mathrm{Cs})$, obtained in an APV experiment at Boulder [23], agrees with the SM value for this quantity and confirms the predicted SM running. However, the interpretation of both the cesium and $\mathrm{NuTeV}$ results has been a subject of debate. For example, the extraction of $Q_{W}(\mathrm{Cs})$ from the experimental PV amplitude relies on intricate atomic structure computations [25-32], and the level of agreement with the SM has varied significantly as additional atomic structure effects have been incorporated in the calculations (see Sec. II for a discussion). Similarly, the NuTeV discrepancy may result from previously unaccounted effects in parton distribution functions $[12,13]$. At present, there are no other determinations of $\sin ^{2} \theta_{W}$ off the $Z$ peak which have comparable precision.

Our discussion of the physics of $Q_{W}(p)$ is organized as follows. In Sec. II, we review some general considerations of the PV ep asymmetry and how $Q_{W}(p)$ is extracted from it. We argue that this will be a theoretically cleaner procedure than the current extraction of $Q_{W}(\mathrm{Cs})$ from APV. Section III gives details of the SM prediction for $Q_{W}(p)$, which provides the baseline for comparison with experiment. Section IV is devoted to the prospective model independent constraints the new $Q_{W}(p)$ experiment would generate. In Secs. $\mathrm{V}$ and VI we analyze the sensitivity of $Q_{W}(p)$ to extra neutral gauge bosons, supersymmetry (SUSY), and leptoquarks (LQs). We summarize our conclusions in Sec. VII.

\section{PARITY VIOLATING $e p$ SCATTERING AND $Q_{W}(p)$}

The PV ep asymmetry has the simple form [33],

$$
A_{L R}=\frac{\sigma_{L}-\sigma_{R}}{\sigma_{L}+\sigma_{R}}=-\frac{G_{F} Q^{2}}{4 \sqrt{2} \pi \alpha}\left[Q_{W}(p)+F^{p}\left(Q^{2}, \theta\right)\right]
$$

where $G_{F}$ is the Fermi constant, $Q^{2}$ is the momentum transfer, and $F^{p}$ is a form factor. At forward angles, one has $F^{p}$ $=Q^{2} B\left(Q^{2}\right)$, where $B\left(Q^{2}\right)$ depends on the nucleon, electromagnetic (EM), and strangeness form factors. The present program of $\mathrm{PV} e p$ scattering experiments-which involve measurements $[19,20,34,35]$ of $A_{L R}$ over a wide kinematic range-is designed to determine $F^{p}$ for forward angles at $Q^{2}$ values as low as $\sim 0.1 \mathrm{GeV}^{2}$. The determination of $Q_{W}(p)$ involves an additional $A_{L R}$ measurement at $Q^{2}$ $\sim 0.03 \mathrm{GeV}^{2}$. Such a value of $Q^{2}$ is optimal for separating $Q_{W}(p)$ from $F^{p}$ with sufficient precision, while retaining sufficient statistics (note that $A_{L R}$ is itself proportional to $Q^{2}$ ). The E-158 experiment is being carried out at almost the same value of $Q^{2}$.
An important feature of the asymmetry in Eq. (2) is its interpretability. Current conservation implies that $Q_{W}(p)$ is protected from large strong interaction corrections involving the low energy structure of the proton. As we note in Sec. III, residual strong interaction corrections involving, e.g., two boson exchange box diagrams, are suppressed at $Q^{2}=0$. Effects that depend on $Q^{2}$ are included in $F^{p}$ and will be constrained by the aforementioned program of experiments, thereby eliminating the need for a first principles nucleon structure calculation. Based on present and future measurements, the extrapolation of $F^{p}$ to $Q^{2}=0$ is expected to induce a $2 \%$ uncertainty and will thus be considered a part of the experimental error budget. ${ }^{1}$

In this respect, the extraction of $Q_{W}(p)$ from $A_{L R}$ is complementary to the recent determination of $Q_{W}(\mathrm{Cs})$ in APV. The latter relies on an advanced atomic theory calculation of the small PV $6 s \rightarrow 7 s$ transition amplitude. Experimentally, the transition amplitude has been measured [23] to a relative precision of $0.35 \%$. Subsequently, by measuring the ratio of the off-diagonal hyperfine amplitude (which is known precisely [36]) to the tensor transition polarizability [37], it was possible to determine $Q_{W}(\mathrm{Cs})$ with a combined experimental and theoretical uncertainty of $0.6 \%$. The result differed by $2.3 \sigma$ from the SM prediction [4] for $Q_{W}(\mathrm{Cs})$. However, updating the corrections from the Breit interaction [25-27] and to a lesser degree from the neutron distribution $[25,38]$ reduced the difference to only $1.0 \sigma$, seemingly removing the discrepancy. Subsequent calculations included other large and previously underestimated contributions (e.g., from QED radiative corrections), some increasing [28$30]$, others decreasing $[31,32]$ the deviation. The atomic theory community now appears to agree on a $0.5 \%$ atomic structure uncertainty for $Q_{W}(\mathrm{Cs})$, and in what follows we adopt the value

$$
Q_{W}(\mathrm{Cs})=-72.69 \pm 0.48 \text {. }
$$

There is also a noteworthy but less precise determination in $\mathrm{Tl}[21,22], Q_{W}(\mathrm{Tl})=-116.6 \pm 3.7$.

A possible strategy for circumventing atomic theory uncertainties is to measure APV for different atoms along an isotope chain. Isotope ratios $\mathcal{R}$ are relatively insensitive to details of the atomic structure and the attendant theoretical uncertainties, making them attractive alternatives to the weak charge of a single isotope as a new physics probe. As shown in Ref. [2], any shift in $\mathcal{R}$ from its SM value due to new physics would be dominated by the change in $Q_{W}(p)$, as the effects on $\mathcal{R}$ of new physics corrections to the weak charge of the neutron, $Q_{W}(n)$, are suppressed. Moreover, $\mathcal{R}$ receives important contributions from changes in the neutron distribution along the isotope chain [38-41]. At present, the corresponding nuclear structure uncertainties seem larger than needed to make $\mathcal{R}$ a useful probe of new physics effects

\footnotetext{
${ }^{1}$ In practice, this extrapolation can be implemented using chiral perturbation theory. Present and future measurements will determine all the relevant low energy constants.
} 
on $Q_{W}(p)$. In contrast, ep scattering will yield $Q_{W}(p)$ without nuclear structure complications.

Given the suppression of $Q_{W}(p)$ in the SM tree level expression (1), a $4 \%$ measurement would provide a theoretically clean probe of new physics with a sensitivity comparable to that achieved by a $0.5 \%$ total error in $Q_{W}(\mathrm{Cs})$, but with entirely different systematical and theoretical uncertainties. Note, however, that measurements of $A_{L R}$ and single isotope APV are complementary as they probe different combinations of the $C_{1 i}$. For example, in contrast to the weak charges of heavy elements, $Q_{W}(p)$ depends significantly on the oblique parameter $T$, introduced in Ref. [42].

\section{III. $Q_{W}(p)$ IN THE STANDARD MODEL}

At tree level in the SM, $Q_{W}(p)$ is given by Eq. (1). Including radiative corrections one can write

$$
\begin{aligned}
Q_{W}(p)= & {\left[\rho_{N C}+\Delta_{e}\right]\left[1-4 \sin ^{2} \hat{\theta}_{W}(0)+\Delta_{e}^{\prime}\right] } \\
& +\square_{W W}+\square_{Z Z}+\square_{\gamma Z} .
\end{aligned}
$$

The parameter $\rho_{N C}=1+\Delta \rho$ [43] renormalizes the ratio of neutral to charged current interaction strengths at low energies, and is evaluated including higher order QCD [44-47] and EW [48-50] corrections. We also include relatively small electron vertex and external leg corrections, which are corrections to the axial-vector Zee and yee couplings, respectively [51],

$$
\Delta_{e}=-\frac{\alpha}{2 \pi}, \quad \Delta_{e}^{\prime}=-\frac{\alpha}{3 \pi}\left(1-4 \hat{s}^{2}\right)\left[\ln \left(\frac{M_{Z}^{2}}{m_{e}^{2}}\right)+\frac{1}{6}\right] .
$$

The latter, which corresponds to the anapole moment of the electron, depends on the choice of EW gauge and is not by itself a physical observable [52]. The purely weak box contributions are given by $[51,53]$

$$
\square_{W W}=\frac{7 \hat{\alpha}}{4 \pi \hat{s}^{2}}, \quad \square_{Z Z}=\frac{\hat{\alpha}}{4 \pi \hat{s}^{2} \hat{c}^{2}}\left(\frac{9}{4}-5 \hat{s}^{2}\right)\left(1-4 \hat{s}^{2}+8 \hat{s}^{4}\right),
$$

where $\hat{\alpha} \equiv \hat{\alpha}\left(M_{Z}\right)$ and $\hat{s}^{2} \equiv 1-\hat{c}^{2} \equiv \sin ^{2} \hat{\theta}_{W}\left(M_{Z}\right)$ are the modified minimal subtraction ( $\overline{\mathrm{MS}}$ ) renormalized QED coupling and the weak mixing angle at the $Z$ scale, respectively. Numerically, the $W W$ box amplitude generates an important $26 \%$ correction to $Q_{W}(p)$, while the $Z Z$ box effect about $3 \%$.

These diagrams are dominated by intermediate states having $p^{2} \sim O\left(M_{W, Z}^{2}\right)$. The corresponding QCD corrections are, thus, perturbative and can be evaluated by relying on the operator product expansion (OPE). At short distances, the product of weak currents entering the hadronic side of the box graphs is equivalent to a series of local operators whose Wilson coefficients can be evaluated by matching with a free field theory calculation. Because the weak (axial) vector current is (partially) conserved, the resulting operators have no anomalous dimensions. Consequently, the perturbative QCD (PQCD) contributions introduce no large logarithms.

In order to evaluate the $O\left(\alpha_{s}\right)$ corrections to these graphs, we follow Ref. [54] where analogous corrections for neutron $\beta$ decay are computed. For the $W W$ box graphs, we have the amplitude

$$
\begin{aligned}
i \mathcal{M}_{W W}= & i\left(\frac{g}{2 \sqrt{2}}\right)^{4} \int \frac{d^{4} k}{(2 \pi)^{4}} \bar{e}\left(K^{\prime}\right) \gamma^{\nu}\left(1-\gamma_{5}\right) \\
& \times k \gamma^{\mu}\left(1-\gamma_{5}\right) e(K) T_{\mu \nu}(k) \frac{1}{k^{2}} \frac{1}{\left(k^{2}-M_{W}^{2}\right)^{2}},
\end{aligned}
$$

where

$$
T_{\mu \nu}(k)=\int d^{4} x e^{-i k \cdot x}\left\langle p^{\prime}\left|T\left(J_{\mu}^{+}(0) J_{\nu}^{-}(x)\right)\right| p\right\rangle,
$$

with $J_{\mu}^{ \pm}(x)$ being the charge changing weak currents. Since the loop integral is infrared finite and is dominated by intermediate states having $k \sim M_{W}$, we have dropped all dependence on $m_{e}$ and the electron momenta $K$ and $K^{\prime}$. The error introduced by this approximation is of order $\left(E_{e} / M_{W}\right)^{2}$ $\sim 0.02 \%$ for the kinematics of the planned experiment, and is negligible for our purposes. A little algebra allows us to rewrite Eq. (7) as

$$
\begin{aligned}
i \mathcal{M}_{W W}= & 2 i\left(\frac{g}{2 \sqrt{2}}\right)^{4} \int \frac{d^{4} k}{(2 \pi)^{4}} \bar{e}\left(K^{\prime}\right)\left[k^{\nu} \gamma^{\mu}+k^{\mu} \gamma^{\nu}-g^{\mu \nu} k\right. \\
& \left.+i \epsilon^{\mu \nu \alpha \lambda} \gamma_{\lambda} \gamma_{5} k_{\alpha}\right]\left(1-\gamma_{5}\right) e(K) T_{\mu \nu}(k) \frac{1}{k^{2}} \\
& \times \frac{1}{\left(k^{2}-M_{W}^{2}\right)^{2}} .
\end{aligned}
$$

The terms proportional to $k^{\mu} T_{\mu \nu}$ and $k^{\nu} T_{\mu \nu}$ are protected from large PQCD corrections by symmetry considerations. This feature may be seen by observing that

$$
\begin{aligned}
k^{\nu} T_{\mu \nu}= & \int d^{4} x\left(i \partial^{\nu} e^{-i k \cdot x}\right)\left\langle p^{\prime}\left|T\left(J_{\mu}^{+}(0) J_{\nu}^{-}(x)\right)\right| p\right\rangle \\
= & i \int d^{4} x e^{-i k \cdot x} \delta\left(x_{0}\right)\left\langle p^{\prime}\left|\left[J_{\mu}^{+}(0), J_{0}^{-}(x)\right]\right| p\right\rangle \\
& -i \int d^{4} x e^{-i k \cdot x}\left\langle p^{\prime}\left|T\left(J_{\mu}^{+}(0) \partial^{\nu} J_{\nu}^{-}(x)\right)\right| p\right\rangle
\end{aligned}
$$

after integration by parts. The divergence $\partial^{\nu} J_{\nu}^{-}(x)$ vanishes in the chiral limit, and in keeping with the high-momentum dominance of the integral, may be safely neglected. On the other hand, the equal time commutator gives $-4 i\left\langle p^{\prime}\left|J_{\mu}^{3}(0)\right| p\right\rangle$, where $J_{\mu}^{3}=\bar{q}_{L} \gamma_{\mu} \tau_{3} q_{L}$ and $q=(u, d)$. Note that the commutator term results from the $S U(2)_{L} \times U(1)_{Y}$ symmetry of the theory, so it is not affected by QCD corrections. 
In contrast, terms involving $T_{\mu}^{\mu}$ and $\epsilon^{\mu \nu \alpha \lambda} T_{\mu \nu}$ cannot be related to equal time commutators and, thus, involve bona fide short distance operator products. In the OPE, the leading local operator appearing in $T_{\mu}^{\mu}$ is just $J_{\mu}^{3}$, whereas for the antisymmetric part, one has the isoscalar current, $J_{\mu}^{I=0}$ $=\bar{q}_{L} \gamma_{\mu} q_{L}$. The leading PQCD contributions to the corresponding Wilson coefficients have been worked out in Refs. [54-56]. For both $T_{\mu}^{\mu}$ and $\epsilon^{\mu \nu \alpha \lambda} T_{\mu \nu}$, the correction factor is $1-\alpha_{s}\left(k^{2}\right) / \pi$. Since the loop integrals are dominated by $k^{2}$ $\sim M_{W}^{2}$, one may approximate the impact on $i \mathcal{M}_{W W}$ by factoring $1-\alpha_{s}\left(M_{W}^{2}\right) / \pi$ out of the corresponding parts of the integral in Eq. (9). The error associated with this approximation is of order $\alpha_{s}^{2}$ and is devoid of any large logarithms. The resulting expression for the $W W$ box contribution to $Q_{W}(p)$ is

$$
\square_{W W}=\frac{\hat{\alpha}}{4 \pi \hat{s}^{2}}\left[2+5\left(1-\frac{\alpha_{s}\left(M_{W}^{2}\right)}{\pi}\right)\right],
$$

where the first term inside the square brackets arises from the equal time commutator. Numerically, the $O\left(\alpha_{s}\right)$ term yields an $\approx-3 \%$ correction to $\square_{W W}$, for an $\approx-0.7 \%$ correction to $Q_{W}(p)$. Higher order PQCD corrections should be an order of magnitude smaller, so the error in $Q_{W}(p)$ associated with truncation at $O\left(\alpha_{s}\right)$ is well below the expected experimental uncertainty.

The calculation of PQCD corrections to $\square_{\text {ZZ }}$ follows along similar lines. In this case, however, all equal time commutators vanish, so that the entire integral carries a 1 $-\alpha_{s}\left(M_{Z}^{2}\right) / \pi$ correction factor. The resulting shift in $Q_{W}(p)$ is $-0.1 \%$, and higher order PQCD effects are negligible. For both $\square_{W W}$ and $\square_{Z Z}$ contributions from lower loop momenta $\left(k^{2} \ll M_{W}^{2}\right)$ are associated with non-perturbative QCD effects. Such contributions, however, carry explicit $\left(p / M_{W, Z}\right)^{2}$ suppression factors, where $p$ is an external momentum or mass. Taking $p \sim E_{e} \sim 1 \mathrm{GeV}$ implies that these non-perturbative contributions are suppressed by at least a few $\times 10^{-4}$, so we may safely neglect them here. A similar conclusion applies to matrix elements of higher order operators in the OPE analysis of $T_{\mu \nu}$ given above.

As a corollary, we have also computed the analogous correction to $Q_{W}(n)$. Again, the $Z Z$ box contribution receives an overall factor, $1-\alpha_{s}\left(M_{Z}^{2}\right) / \pi$, while for the $W W$ box we obtain

$$
\square_{W W}^{(n)}=\frac{\hat{\alpha}}{4 \pi \hat{s}^{2}}\left[-2+4\left(1-\frac{\alpha_{s}\left(M_{W}^{2}\right)}{\pi}\right)\right] .
$$

Notice that the sum of Eqs. (11) and (12) is also corrected by an overall factor, $1-\alpha_{s}\left(M_{W}^{2}\right) / \pi$, as is expected from an isoscalar combination where no equal time commutator should be involved. The resulting shifts in the SM predictions for $Q_{W}(\mathrm{Cs})$ and $Q_{W}(\mathrm{Tl})$ are -0.07 and -0.11 , respectively, or $+0.1 \%$.

In contrast, the $\gamma Z$ box contribution,

$$
\square_{\gamma Z}=\frac{5 \hat{\alpha}}{2 \pi}\left(1-4 \hat{s}^{2}\right)\left[\ln \left(\frac{M_{Z}^{2}}{\Lambda^{2}}\right)+C_{\gamma Z}(\Lambda)\right],
$$

contains some sensitivity to the low momentum regime. The scale $\Lambda \sim O(1 \mathrm{GeV})$ appearing here denotes a hadronic cutoff associated with the transition between short and long distance contributions to the loop integral. The former are calculable and are dominated by the large logarithm $\ln M_{Z}^{2} / \Lambda^{2}$. At present, however, one cannot compute long distance contributions from first principles in QCD. Consequently, we parametrize them by the constant $C_{\gamma Z}(\Lambda)$, whose $\Lambda$ dependence must cancel that associated with the short distance logarithm. We note that a similar situation arises in radiative corrections to $G_{V}$ in neutron and nuclear $\beta$ decay, where the $\gamma W$ box diagram contains a short distance logarithm and a presently uncalculable long distance term $C_{\gamma W}(\Lambda)$.

In the case of $Q_{W}(p)$, the uncertainty associated with $C_{\gamma Z}(\Lambda)$ is suppressed by the $\left(1-4 \hat{s}^{2}\right)$ prefactor $^{2}$ in Eq. (13). This factor arises from the sum of box and crossed-box diagrams, leading to an antisymmetric product of the lepton EM and weak neutral currents [2,51]. Since the resulting leptonic part of the box amplitude must be axial vector in character, only the vector part of the weak neutral current of the electron enters which is proportional to $1-4 \hat{s}^{2}$. This result is quite general and independent of the hadronic part of the diagram. To estimate this uncertainty numerically, we follow Ref. [57] setting $\Lambda=m_{\rho}$ and $C_{\gamma Z}\left(m_{\rho}\right)=3 / 2 \pm 1$, which translates into a $\pm 0.65 \%$ uncertainty in $Q_{W}(p)$. The central value for $C_{\gamma Z}\left(m_{\rho}\right)$ is obtained from a free quark calculation. A more detailed analysis, taking into account contributions from intermediate excited states of the proton, is likely to shift $C_{\gamma Z}$, but we do not expect the change to be considerably larger than the estimated uncertainty. In any case, increasing the error bar on $C_{\gamma Z}$ by a factor of 5 would still imply an uncertainty in $Q_{W}(p)$ below the expected experimental error. For comparison, we note that a change in the value of $C_{\gamma W}(\Lambda)$ of similar magnitude would substantially affect the extraction of $\left|V_{u d}\right|^{2}$ from light quark $\beta$ decays, causing the first row of the CKM matrix to deviate from unitarity by several standard deviations. Since the dynamics entering $C_{\gamma Z}$ and $C_{\gamma W}$ are similar, it appears unlikely that the uncertainty in $C_{\gamma Z}$ could differ significantly from \pm 1 .

The remaining hadronic contribution to $Q_{W}(p)$ arises from the low energy weak mixing angle $\sin ^{2} \hat{\theta}_{W}(0)$, which is the EW analog of the EM coupling $\hat{\alpha}$. The latter is measured very precisely in the Thomson limit $\left(q^{2}=0\right)$, but hadronic contributions induce a sizable uncertainty for large $q^{2}$, and most importantly for $q^{2}=M_{Z}^{2}$ [58]. Conversely, $\hat{s}^{2}$ is measured precisely at the $Z$ pole, but hadronic loops induce an uncertainty for $q^{2}=0$, which is correlated but not identical to the one in $\hat{\alpha}$. Note that effects due to $q^{2} \neq 0$ are already taken into account experimentally via the $Q^{2}$ expansion and extrapolation of $F^{p}$ (see Sec. II). One can then define

\footnotetext{
${ }^{2}$ Additional contributions arise that are not suppressed by this factor, but are negligible because they go as $\left(E_{e} / M_{Z}\right)^{2}$.
} 


$$
\begin{aligned}
\sin ^{2} \hat{\theta}_{W}(0)= & \hat{s}^{2}+\Delta \kappa_{\text {had }}^{(5)} \\
& +\frac{\alpha}{\pi}\left\{\frac { ( 1 - 4 \hat { s } ^ { 2 } ) } { 1 2 } \left[\sum_{\ell} \ln \left(\frac{M_{Z}^{2}}{m_{\ell}^{2}}\right)\left(1+\frac{3 \alpha}{4 \pi}\right)\right.\right. \\
& \left.\left.+\frac{135 \alpha}{32 \pi}\right]-\left[\frac{7 \hat{c}^{2}}{4}+\frac{1}{24}\right] \ln \left(\frac{M_{Z}^{2}}{M_{W}^{2}}\right)+\frac{\hat{s}^{2}}{6}-\frac{7}{18}\right\},
\end{aligned}
$$

where the sum is over the charged leptons, and we find, for the hadronic contribution,

$$
\Delta \kappa_{\mathrm{had}}^{(5)}=(7.90 \pm 0.05 \pm 0.06) \times 10^{-3}
$$

inducing a $0.4 \%$ uncertainty in $Q_{W}(p)$. The first error in Eq. (15) is correlated with the uncertainty in $\Delta \hat{\alpha}_{\mathrm{had}}^{(5)}\left(M_{Z}^{2}\right)$ [59]. The second error is from the conversion of $\Delta \kappa_{\text {had }}^{(5)}$ which induces an uncertainty from the flavor separation of the $e^{+} e^{-}$annihilation and $\tau$ decay data. This updates the value in Ref. [57], $\Delta \kappa_{\mathrm{had}}^{(5)}=(7.96 \pm 0.58) \times 10^{-3}$. Note that the uncertainty in $\Delta \kappa_{\text {had }}^{(5)}$ is also related to the vacuum polarization uncertainty $[10,11]$ in $a_{\mu}$. These correlations should be properly treated in global analyzes of precision data. With $\hat{s}^{2}=0.23112 \pm 0.00015$ from a SM fit to all current data, Eqs. (4) and (14) yield

$$
\begin{aligned}
\sin ^{2} \hat{\theta}_{W}(0) & =0.23807 \pm 0.00017 \\
Q_{W}(p) & =0.0716 \pm 0.0006
\end{aligned}
$$

where the uncertainty in the prediction for $Q_{W}(p)$ is from the input parameters and dominated by the error in $\hat{s}^{2}$. The latter will decrease significantly in the future [60]. Taken together, the hadronic effects arising from $\Delta \kappa_{\text {had }}^{(5)}$ and the box graphs combine to give a theoretical uncertainty of $0.8 \%$.

The QWEAK experiment [14] seeks to perform the most precise determination of the weak mixing angle off the $Z$ pole. For example, a $4 \%$ determination $\Delta Q_{W}(p)= \pm 0.0029$ [14] (assuming a $2.8 \%$ statistical plus $2.8 \%$ systematic plus $0.8 \%$ theoretical error) would yield an uncertainty

$$
\Delta \sin ^{2} \hat{\theta}_{W}(0)= \pm 7.2 \times 10^{-4} .
$$

While the precise definition of $\sin ^{2} \hat{\theta}_{W}(0)$ is scheme dependent, this quantity is nonetheless useful for comparing different low energy experiments. Furthermore, as illustrated in Fig. 1, the $q^{2}$ evolution from the $Z$ pole as predicted by the SM,

$$
\sin ^{2} \hat{\theta}_{W}(0)-\hat{s}^{2}=0.00694 \pm 0.00074
$$

could be established with more than 9 standard deviations. For comparison, the cleanest test of PQCD can be obtained by contrasting the $\tau$ lepton lifetime with the hadronic $Z$ decay width: when interpreted as the RGE evolution of $\alpha_{s}$ from $m_{\tau}$ to $M_{Z}$, the result of the latest analysis [61] corresponds to an $11 \sigma$ effect.

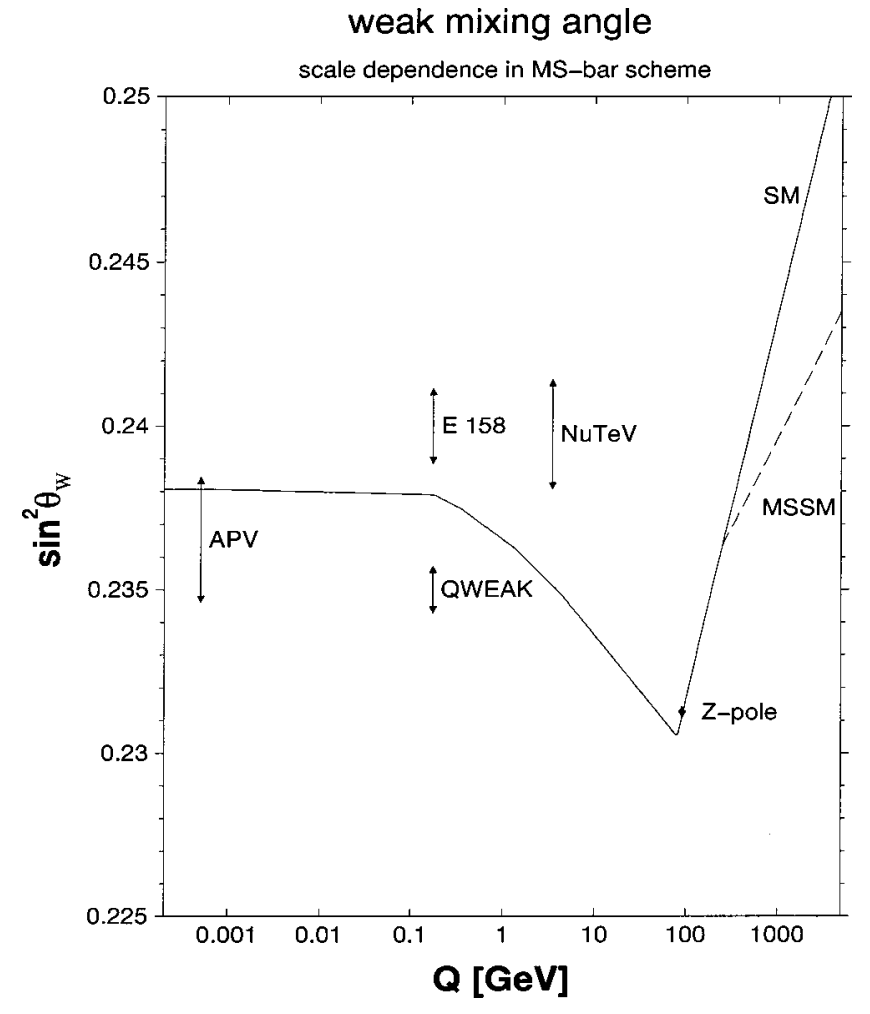

FIG. 1. Calculated running of the weak mixing angle in the SM, defined in the $(\overline{\mathrm{MS}})$ renormalization scheme (the dashed line indicates the reduced slope typical for the minimal supersymmetric standard model). Shown are the results from APV (Cs and $\mathrm{Tl}$ ), $\mathrm{NuTeV}$, and the $Z$ pole. QWEAK and E 158 refer to the future $Q_{W}(p)$ and $Q_{W}(e)$ measurements and have arbitrarily chosen vertical locations.

Before proceeding, we comment on one additional possible source of hadronic effects in $Q_{W}(p)$ : isospin admixtures in the proton wave function. The SM value quoted above implicitly assumes that the proton is an exact eigenstate of isospin. The EM and weak neutral vector currents for light quarks can then be decomposed according to their isospin content,

$$
\begin{aligned}
J_{\mu}^{E M} & =\sum_{q=u, d} Q_{q} \bar{q} \gamma_{\mu} q=J_{\mu}^{I=1}+J_{\mu}^{I=0}, \\
J_{\mu}^{N C} & =-2 \sum_{q=u, d} C_{1 q} \bar{q} \gamma_{\mu} q \\
& =-2\left(C_{1 u}-C_{1 d}\right) J_{\mu}^{I=1}-6\left(C_{1 u}+C_{1 d}\right) J_{\mu}^{I=0},
\end{aligned}
$$

where the $C_{1 q}$ are defined in Eq. (26). For the purpose of this discussion, we neglect contributions from strange quarks, which are effectively contained in $F^{p}$ term in Eq. (2). To the extent to which the nucleon is a pure $I=1 / 2$ isospin eigenstate, one has $F_{1}^{p}(0)^{I=1}=F_{1}^{p}(0)^{I=0}=1 / 2$, where the $F_{1}^{p}(0)^{I}$ are the Dirac form factors associated with the proton matrix elements of the $J_{\mu}^{I}$. In principle, these form factor relations receive small corrections due to isospin breaking light quark mass differences $\left(m_{u} \neq m_{d}\right)$ and EM effects. However, con- 
servation of EM charge implies that such corrections vanish. To see this, assume that the proton state contains a small, $O(\epsilon)$, admixture of an $I^{\prime} \neq 1 / 2$ state

$$
|p\rangle=\sqrt{1-\epsilon^{2}}|1 / 2,1 / 2\rangle+\epsilon\left|I^{\prime}, I_{3}^{\prime}\right\rangle,
$$

where, for the purpose of this illustration, we drop explicit $O\left(\epsilon^{2}\right)$ terms involving the $\left|I^{\prime}, I_{3}^{\prime}\right\rangle$ state. At $q^{2}=0$, the charges $J_{0}^{I}$ are equivalent to the operators $\hat{I}_{3}$ and $\frac{1}{2} \hat{1}$. Since these operators cannot connect states of different total isospin, one has

$$
\begin{aligned}
& F_{1}^{p}(0)^{I=1}=\frac{1}{2}\left(1-\epsilon^{2}\right)+\epsilon^{2} I_{3}^{\prime}, \\
& F_{1}^{p}(0)^{I=0}=\frac{1}{2} .
\end{aligned}
$$

Since the proton charge is $1=F_{1}^{p}(0)^{I=1}+F_{1}^{p}(0)^{I=0}$, one must have $I_{3}^{\prime}=1 / 2$, so that there are no corrections to $F_{1}^{p}(0)^{I}$ through $O\left(\epsilon^{2}\right)$. Thus, one has to this order for the neutral current Dirac form factor,

$$
Q_{W}(p) \equiv F_{1}^{p}(0)^{N C}=-2\left(2 C_{1 u}+C_{1 d}\right),
$$

which is the same result obtained in the absence of any isospin impurities. Similar arguments prevent the appearance of any higher order terms in $\epsilon$.

\section{FOUR-FERMI OPERATORS AND MODEL INDEPENDENT ANALYSIS}

Before considering the consequences for particular models of new physics, it is instructive to consider the model independent implications of a $4 \% Q_{W}(p)$ measurement. The low energy effective electron-quark Lagrangian of the form $A(e) \times V(q)$ is given by

$$
\mathcal{L}=\mathcal{L}_{\mathrm{SM}}^{\mathrm{PV}}+\mathcal{L}_{\mathrm{NEW}}^{\mathrm{PV}}
$$

where

$$
\begin{gathered}
\mathcal{L}_{\mathrm{SM}}^{\mathrm{PV}}=-\frac{G_{F}}{\sqrt{2}} \bar{e} \gamma_{\mu} \gamma_{5} e \sum_{q} C_{1 q} \bar{q} \gamma^{\mu} q, \\
\mathcal{L}_{N E W}^{\mathrm{PV}}=\frac{g^{2}}{4 \Lambda^{2}} \bar{e} \gamma_{\mu} \gamma_{5} e \sum_{f} h_{V}^{q} \bar{q} \gamma^{\mu} q,
\end{gathered}
$$

and where $g, \Lambda$, and $h_{V}^{q}$ are, respectively, the coupling constant, the mass scale, and effective coefficients associated with the new physics. ${ }^{3}$ The latter are in general of order unity; the explicit factor of 4 arises from the projection operators on left and right (or vector and axial-vector) chiral fermions. In the same normalization, the SM coefficients take the values (see Ref. [4]) $C_{1 u} / 2=-0.09429 \pm 0.00011$ and $C_{1 d} / 2=+0.17070 \pm 0.00007$ for up and down quarks,

\footnotetext{
${ }^{3}$ The couplings $C_{2 q}$ are defined as in Eq. (26) with $\gamma_{5}$ appearing between the quark fields instead of the electron fields.
}

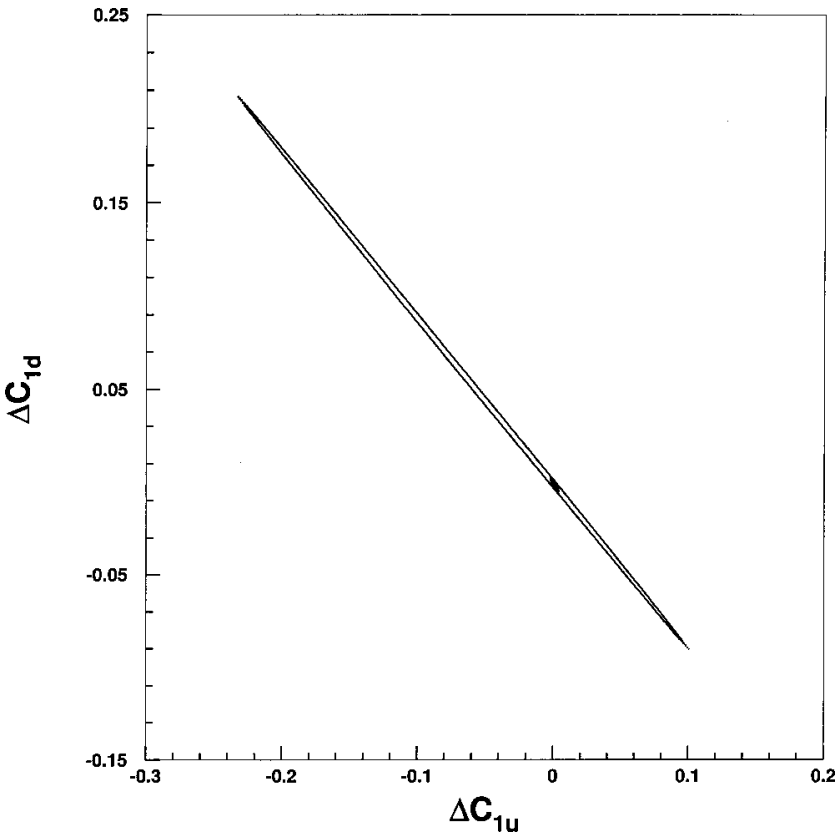

FIG. 2. Present and prospective $90 \%$ C.L. constraints on new physics contributions to the $e q$ couplings $C_{1 u}$ and $C_{1 d}$. The larger ellipse represents the present constraints, derived from APV in Cs [23], and polarized electron scattering at MIT-Bates [18] and SLAC [15]. The smaller ellipse indicates the constraints after the inclusion of the $Q_{W}(p)$ measurement, assuming that the central experimental value coincides with the SM prediction.

respectively, where we included the QCD corrections obtained in Eqs. (11) and (12), and where the uncertainties are from the SM inputs. We find

$$
Q_{W}^{p}(\mathrm{SM})=-2\left(2 C_{1 u}+C_{1 d}\right)=0.0716 \pm 0.0006 .
$$

A $4 \%$ measurement of $Q_{W}(p)$ would thus test new physics scales up to

$$
\frac{\Lambda}{g} \approx{\frac{1}{\left(\sqrt{2} G_{F}\left|\Delta Q_{W}^{p}\right|\right)}}^{1 / 2} \approx 4.6 \mathrm{TeV} .
$$

The sensitivity to non-perturbative theories (such as technicolor, models of composite fermions, or other strong coupling dynamics) with $g \sim 2 \pi$ could even reach $\Lambda \approx 29 \mathrm{TeV}$. As another example, for extra $Z^{\prime}$ bosons from simple models based on grand unified theories (GUT), one expects $g$ $\sim 0.45$, so that one can study such bosons (with unit charges) up to masses $M_{Z^{\prime}} \approx 2.1 \mathrm{TeV}$. $Z^{\prime}$ bosons are predicted in many extensions of the SM ranging from the more classical GUT and technicolor models to SUSY and string theories. We discuss the sensitivity of $Q_{W}(p)$ to $Z^{\prime}$ bosons, as well as other scenarios, in the subsequent sections.

In Fig. 2 we plot the present constraints on $\Delta C_{1 u}$ and $\Delta C_{1 d}$, the shifts in the $C_{1 q}$ caused by new physics. They are derived from $Q_{W}(\mathrm{Cs})$ [23], as well as the MIT-Bates ${ }^{12} \mathrm{C}$ [18] and SLAC deuterium [15] parity violation measurements. As long as $\Delta C_{1 u}$ and $\Delta C_{1 d}$ are almost perfectly correlated, the result is an elongated ellipse. The impact of the proposed $Q_{W}(p)$ measurement is indicated by the smaller 


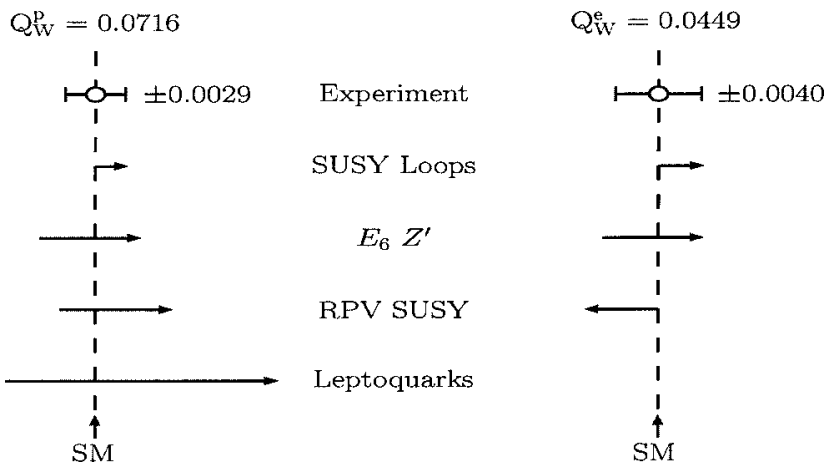

FIG. 3. Comparison of anticipated errors for $Q_{W}(p)$ and $Q_{W}(e)$ with deviations from the SM expected from various extensions and allowed (at $95 \%$ C.L.) by fits to existing data. Note that the two measurements are highly complementary. They would shift in a strongly correlated manner due to SUSY loops or a $(1 \mathrm{TeV}) Z^{\prime}$ and thus together they could result in evidence for such new physics. In the case of RPV SUSY, the two measurements are somewhat anticorrelated. Finally, only $Q_{W}(p)$ is sensitive to LQs, while $Q_{W}(e)$ would serve as a control.

ellipse. The dramatic reduction in the allowed parameter space will be possible because $Q_{W}(p)$ probes a very different linear combination than the existing data.

In the next two sections we turn to specific extensions of the SM, of which there are many, and focus on three particularly well motivated types: gauge bosons, SUSY, and LQs. In doing so, we emphasize on the complementarity of the PV Møller asymmetry measured by the SLAC-E-158 experiment [24] which has comparable anticipated precision and (as a purely leptonic observable) has a clean theoretical interpretation. Some new physics scenarios appear more strongly in the semileptonic channel than in the purely leptonic channel and vice versa. The complementarity of the two measurements is advantageous in attempting to distinguish among various new physics scenarios and is summarized in Fig. 3.

\section{EXTRA NEUTRAL GAUGE INTERACTIONS}

The introduction of neutral gauge symmetries beyond those associated with the photon and the $Z$ boson have long been considered as one of the best motivated extensions of the SM. Such $U(1)^{\prime}$ symmetries are predicted in most GUTs and appear copiously in superstring theories. In the context of SUSY, they do not spoil the approximate gauge coupling unification predicted by the simplest and most economic scenarios. Moreover, in many SUSY models [though not the simplest $S O(10)$ ones], the enhanced $U(1)^{\prime}$ gauge symmetry forbids an elementary bilinear Higgs $\mu$ term, while allowing an effective $\mu$ to be generated at the scale of $U(1)^{\prime}$ breaking without introducing cosmological problems [62]. In various string motivated models of radiative breaking, this scale is comparable to the EW scale (i.e., $\lesssim 1 \mathrm{TeV}$ ) $[62,63]$, thereby providing a solution [64] to the $\mu$ problem [65] and enhancing the prospects that a $Z^{\prime}$ could be in reach in collider experiments or seen indirectly in the precision EW data. An extra $U(1)^{\prime}$ symmetry could also explain proton stability, which is not automatic in supersymmetric models, or it could solve both the proton lifetime puzzle and the $\mu$ problem simultaneously [66].

From a phenomenological standpoint, direct searches at the Tevatron [67] have as yet yielded no evidence ${ }^{4}$ for the existence of an extra neutral $Z^{\prime}$ boson associated with the $U(1)^{\prime}$, providing instead only lower bounds of about 600 $\mathrm{GeV}$ (depending on the precise nature of the $Z^{\prime}$ ). This implies a hierarchy of an order of magnitude between the $Z$ and $Z^{\prime}$ masses. Recently, using approximately flat directions in moduli space, it was shown that such a hierarchy can arise naturally in SUSY models [69].

On the other hand, several indirect effects could be attributed to a $Z^{\prime}$. The $Z$ line shape fit at LEP [70] yields a significantly larger value for the hadronic peak cross section $\sigma_{\text {had }}^{0}$ than is predicted in the SM. This implies, for example, that the effective number of massless neutrinos, $N_{\nu}$, is $2.986 \pm 0.007$, which is $2 \sigma$ lower than the SM prediction, $N_{\nu}=3$. As a consequence, the $Z$ pole data currently favors $Z^{\prime}$ scenarios with a small amount of $Z-Z^{\prime}$ mixing $(\sin \theta$ $\neq 0)[71]$ which mimics a negative contribution to the invisible $Z$ decay width. The result by the NuTeV Collaboration [7] can be brought into better agreement when one allows a $Z^{\prime}$, especially when family non-universal couplings are assumed $[71,72]$.

To analyze the impact of a $Z^{\prime}$ on $Q_{W}(p)$, we employ Eq. (27) with $\Lambda=M_{Z^{\prime}}$ and $g=g_{Z^{\prime}}=\sqrt{5 / 3} \sin \theta_{W} \sqrt{\lambda} g_{Z} \quad$ [73], where $\lambda=1$ in the simplest models. $g_{Z}^{2}=\sqrt{32} G_{F} M_{Z}^{2}$ is the SM coupling constant for the ordinary $Z$. Consider the Abelian subgroups of the $E_{6}$ GUT group,

$$
\begin{aligned}
E_{6} & \rightarrow S O(10) \times U(1)_{\psi} \rightarrow S U(5) \times U(1)_{\chi} \times U(1)_{\psi} \\
& \rightarrow S U(3)_{C} \times S U(2)_{L} \times U(1)_{Y} \times U(1)_{\chi} \times U(1)_{\psi} .
\end{aligned}
$$

The most general $Z^{\prime}$ boson from $E_{6}$ can be written as the linear combination [71]

$$
Z^{\prime} \sim-\cos \alpha \cos \beta Z_{\chi}+\sin \alpha \cos \beta Z_{Y}-\sin \beta Z_{\psi} \text {. }
$$

Considerations of gauge anomaly cancellation as well as the proton lifetime and $\mu$ problems in SUSY models mentioned earlier, also favor a $Z^{\prime}$ of that type [66]. The assignment of SM fermions to representations of $S O(10)$ implies that $Z_{\psi}$ has only axial-vector couplings and can generate no PV ef interactions of the type in Eq. (27), whereas the $Z_{\chi}$ generates only PV ed and $e e$ interactions of this type. Moreover, unlike in most other classes of models, the contributions to the weak charges of the proton and the electron would have equal magnitude. Thus, should $Q_{W}(p)$ show a deviation from the SM prediction, a comparison with $Q_{W}(e)$ would be a powerful tool to discriminate between $Z^{\prime}$ and other SM extensions. This statement is illustrated in Fig. 3 where the sensitivities of $Q_{W}(p)$ and $Q_{W}(e)$ are contrasted.

If $Z^{\prime}$ were detected at the Tevatron or the CERN Large Hadron Collider (LHC), it would be important to constrain

\footnotetext{
${ }^{4}$ See, however, Ref. [68] which reports a $2 \sigma$ deficit in the highest mass bin of the leptonic forward-backward asymmetry seen by the Collider Detector at Fermilab (CDF) Collaboration.
} 


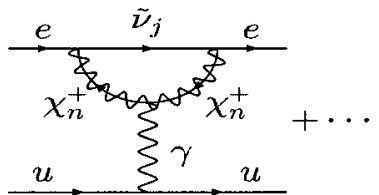

(a)

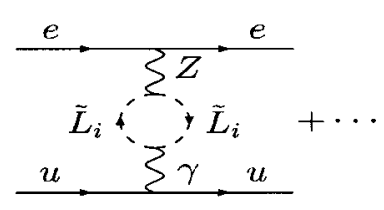

(b)

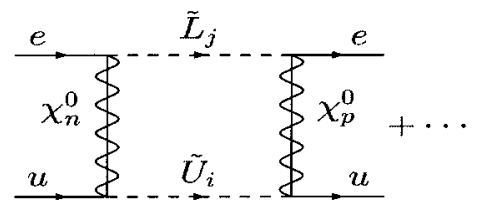

(c)

FIG. 4. Representative examples of SUSY loop corrections to $Q_{W}(p)$. Shown are corrections from (a) charginos and sneutrinos; (b) sleptons contributing to $\gamma-Z$ mixing $\left[\Delta \sin ^{2} \hat{\theta}_{W}(0)_{S U S Y}\right]$; and (c) a box graph containing neutralinos, sleptons, and squarks.

its properties. Its mass would be measured in course of the discovery, while $\sin \theta$ is mainly constrained by LEP 1 . The $U(1)^{\prime}$ charges and the couplings to quarks and leptons, however, are best determined by low energy precision measurements. Currently, the best fit values are $\alpha=-0.8_{-1.2}^{+1.4}, \beta$ $=1.0_{-0.8}^{+0.4}$, and $\sin \theta=0.0010_{-0.0006}^{+0.0012}$, obtained for $\lambda=1$ and $M_{Z^{\prime}}=1 \mathrm{TeV}$. In this case, $Q_{W}(p)=0.0747$ is predicted, i.e., a $1.1 \sigma$ effect. The impact of the QWEAK measurement would be to reduce the allowed region of the parameters $\alpha$ and $\beta$ by $\sim 30 \%$.

\section{SUPERSYMMETRY AND LEPTOQUARKS}

As in the case of extended gauge symmetry, the theoretical motivation for supersymmetric extensions of the SM is strong. SUSY is a prediction of superstring theories; and if the SUSY breaking scale is at the EW scale, it stabilizes the latter and is consistent with coupling unification. Conversely, minimal SUSY introduces a new set of issues, including the scale of the $\mu$ parameter mentioned above and the presence of 105 parameters [74,75] in the soft SUSY breaking Lagrangian. In order to be predictive, additional theoretical constraints must be invoked, such as those provided by gauge, gravity, or anomaly mediated SUSY breaking models. The phenomenological evidence for SUSY thus far is sparse, though hints exist. For example, the neutralino is a natural candidate for cold dark matter, and the possible deviation of $a_{\mu}$ points suggestively toward SUSY. Since, in the end, the experiment will determine what form of SUSY (if any) is applicable to EW phenomena, it is of interest to discuss the prospective implications of a $Q_{W}(p)$ measurement for this scenario.

While baryon number $B$ and lepton number $L$ are exact symmetries of the SM, they are not automatically conserved in the minimal supersymmetric standard model (MSSM). In order to avoid proton decay, $B$ and $L$ conservation-in the guise of $R$ parity conservation-is often imposed by hand. In this case, every MSSM vertex contains an even number of superpartners, and the effects of SUSY appear in $Q_{W}(p)$ only via loops, such as those shown in Fig. 4. Recently, such loop corrections to a variety of low and medium energy precision observables were computed in Refs. [76-78]. These analyses were completed without invoking any assumptions about the mechanism for soft SUSY breaking. The implications of charged current data for the SUSY spectrum appear to conflict with those derived from typical models for SUSY breaking mediation [76]. This conflict may be alleviated by allowing for $R$ parity violation (RPV) [3], though doing so would eliminate the lightest neutralino as a dark matter candidate. From this perspective, independent low energy probes of the MSSM spectrum take on added importance.

A measurement of $Q_{W}(p)$, when considered in tandem with $Q_{W}(e)$ and $Q_{W}(\mathrm{Cs})$, could provide such a probe. The MSSM loop corrections to the weak charges can be analyzed efficiently by modifying Eq. (4),

$$
\begin{gathered}
Q_{W}(p)=\left[\rho_{N C}+\Delta_{e}+\Delta \rho_{\mathrm{SUSY}}\right]\left[1-4 \sin ^{2} \hat{\theta}_{W}(0)+\Delta_{e}^{\prime}\right] \\
+\square_{W W}+\square_{Z Z}+\square_{\gamma Z}+\lambda_{\mathrm{SUSY}}, \\
\sin ^{2} \hat{\theta}_{W}(0)=\sin ^{2} \hat{\theta}_{W}(0)_{\mathrm{SM}}+\Delta \sin ^{2} \hat{\theta}_{W}(0)_{\mathrm{SUSY}},
\end{gathered}
$$

where $\sin ^{2} \hat{\theta}_{W}(0)_{S M}$ is the SM prediction given in Eq. (14) and $\Delta \sin ^{2} \hat{\theta}_{W}(0)_{\text {SUSY }}$ is the correction induced by SUSY loops. ${ }^{5}$ All SUSY box graph contributions, as well as non-universal vertex and external leg corrections, are contained in $\lambda_{\text {SUSY }}$. Flavor-independent corrections are given by $\Delta \rho_{\text {SUSY }}$ and $\Delta \sin ^{2} \hat{\theta}_{W}(0)_{\text {SUSY }}$.

The effects of SUSY loops on $Q_{W}(p)$ and $Q_{W}(e)$ are dominated by $\Delta \sin ^{2} \hat{\theta}_{W}(0)_{\text {SUSY }}$, because present bounds on the $T$ parameter from precision data [4] limit the magnitude of $\Delta \rho_{\text {SUSY }}$. Moreover, box graph contributions are numerically small, while cancellations reduce the impact of vertex and external leg corrections. Consequently, the shifts in the proton and electron weak charges are similar over nearly all allowed SUSY parameter space. This is in contrast to $Q_{W}(\mathrm{Cs})$ due to canceling the corrections to $u$ and $d$ quark weak charges. Thus, should the QWEAK and SLAC E-158 experiments observe a correlated deviation, and should $Q_{W}(\mathrm{Cs})$ remain in agreement with the SM, the MSSM would be a favored explanation compared to many other scenarios.

The situation changes considerably in the presence of RPV effects. The most general gauge invariant, renormalizable RPV extension of the MSSM is generated by the superpotential [79]

$$
W_{R P V}=\frac{1}{2} \lambda_{i j k} L^{i} L^{j} \bar{e}^{k}+\lambda_{i j k}^{\prime} L^{i} Q^{j} \bar{d}^{k}+\frac{1}{2} \lambda_{i j k}^{\prime \prime} \bar{u}^{i} \bar{d}^{j} \bar{d}^{k}+\mu_{i}^{\prime} L^{i} H_{u},
$$

where $L^{i}$ and $Q^{i}$ denote the left-handed lepton and quark doublet superfields, respectively; the barred quantities denote the right-handed singlet superfields; $H_{u}$ is the hypercharge $Y=1$ Higgs superfield; and the indices indicate generations. The bulk of studies of $W_{R P V}$ have been phenomenological

\footnotetext{
${ }^{5}$ In the notation of Ref. [77], $\Delta \sin ^{2} \hat{\theta}_{W}(0)_{\text {SUSY }}=4 \hat{s}^{2} \delta \kappa_{P V}^{\text {susy }}$.
} 


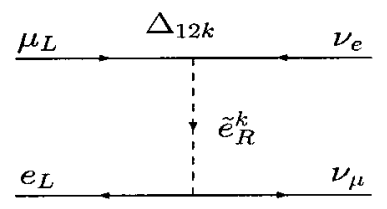

(a)

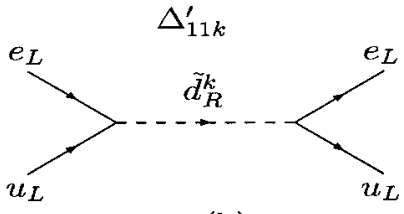

(b)
FIG. 5. Representative examples of tree level SUSY corections in the case of RPV. Shown are (a) a contribution to $\mu$ decay which affects $Q_{W}(p)$ through a modification of $G_{F}$ and $\sin ^{2} \theta_{W}$, and (b) squark exchange.

[80]. The strongest constraint comes from the proton lifetime, which generally forbids the $B$ violating $\lambda^{\prime \prime}$ terms unless all other ( $L$ violating) terms in $W_{R P V}$ vanish. Consequently, we restrict our attention to $\lambda_{i j k}^{\prime \prime}=0$ and, for simplicity, we also set $\mu_{i}^{\prime}=0$. When inserted into the amplitudes of Fig. 5, the remaining interactions in Eq. (32) generate corrections in terms of the quantities $\Delta_{i j k}(\widetilde{f})$ and $\Delta_{i j k}^{\prime}(\widetilde{f})$, where, for example,

$$
\Delta_{12 k}\left(\tilde{e}_{R}^{k}\right)=\frac{\left|\lambda_{12 k}\right|^{2}}{4 \sqrt{2} G_{F} M_{\tilde{e}_{R}^{k}}^{2}},
$$

with $\tilde{e}_{R}^{k}$ being the exchanged slepton, and where the $\Delta_{i j k}^{\prime}(\tilde{f})$ are defined similarly by replacing $\lambda_{i j k} \rightarrow \lambda_{i j k}^{\prime}$. One obtains tree level contributions to $Q_{W}(p)$ such as those shown in Fig. 5. Similar corrections affect other EW observables, such as $Q_{W}(e), Q_{W}(\mathrm{Cs})$, and $G_{V}$. Specifically [3],

$$
\begin{aligned}
\Delta Q_{W}(p) / Q_{W}(p) \approx & \left(\frac{2}{1-4 \sin ^{2} \theta_{W}}\right)\left[-2 \lambda_{x} \Delta_{12 k}\left(\tilde{e}_{R}^{k}\right)\right. \\
& \left.+2 \Delta_{11 k}^{\prime}\left(\tilde{d}_{R}^{k}\right)-\Delta_{1 j 1}^{\prime}\left(\tilde{q}_{L}^{j}\right)\right], \\
\Delta Q_{W}(e) / Q_{W}(e) \approx & -\left(\frac{4}{1-4 \sin ^{2} \theta_{W}}\right) \lambda_{x} \Delta_{12 k}\left(\tilde{e}_{R}^{k}\right),
\end{aligned}
$$

where $\lambda_{x}=\hat{s}^{2} \hat{c}^{2} /\left(1-2 \hat{s}^{2}\right) \approx 0.33$. In contrast to MSSM loop effects, $Q_{W}(p)$ and $Q_{W}(e)$ display complementary sensitivities to RPV effects. To illustrate, we consider a multiparameter fit to precision data, allowing $\Delta_{12 k}, \Delta_{11 k}^{\prime}, \Delta_{1 j 1}^{\prime}$, and $\Delta_{21 k}^{\prime}$ to be non-zero. The results imply that the possible shifts in $Q_{W}(p)$ and $Q_{W}(e)$ have opposite relative signs over nearly all the presently allowed parameter space. We find that shifts of the order $\Delta Q_{W}(p) / Q_{W}(p) \sim 10 \%$ are allowed at the $95 \%$ C.L. Thus, a comparison of $Q_{W}(p)$ and $Q_{W}(e)$ could help distinguish between the versions of SUSY with and without RPV.

The effects of $\lambda^{\prime} \neq 0$ are similar to those generated by scalar LQs. While RPV SUSY provides a natural context in which to discuss the latter, vector LQs arise naturally in various GUT models [81,82]. Assuming $S U(3)_{C} \times S U(2)_{L} \times U(1)_{Y}$ invariance one obtains the Lagrangian [83]

$$
\begin{aligned}
\mathcal{L}= & h_{2}^{L} \bar{u} \ell R_{2}^{L}+h_{2}^{R} \bar{q} i \tau_{2} e R_{2}^{R}+\widetilde{h}_{2} \bar{d} \ell \widetilde{R}_{2}^{L}+g_{1}^{L} \bar{q}^{c} i \tau_{2} \ell S_{1}^{L} \\
& +g_{1}^{R} \bar{u}^{c} e S_{1}^{R}+\widetilde{g}_{1} \bar{d}^{c} e \widetilde{S}_{1}^{R}+g_{3} \bar{q}^{c} i \tau_{2} \vec{\tau} \ell S_{3}+h_{1}^{L} \bar{q} \gamma^{\mu} \ell U_{1 \mu}^{L} \\
& +h_{1}^{R} \bar{d} \gamma^{\mu} e U_{1 \mu}^{R}+\widetilde{h}_{1} \bar{u} \gamma^{\mu} e \widetilde{U}_{1 \mu}^{R}+h_{3} \bar{q} \gamma^{\mu} \vec{\tau} \ell U_{3 \mu} \\
& +g_{2}^{L} \bar{d}^{c} \gamma^{\mu} \ell V_{2 \mu}^{L}+g_{2}^{R} \bar{q}^{c} \gamma^{\mu} e V_{2 \mu}^{R}+\tilde{g}_{2} \bar{u}^{c} \gamma^{\mu} \ell \widetilde{V}_{2 \mu}^{L}+\text { H.c. }
\end{aligned}
$$

where $q$ and $l$ and the left-handed quark and lepton doublets and $u, d$, and $e$ are the right-handed singlets. Since we are interested in the implications for $Q_{W}(p)$, we only consider first generation LQs. The first two rows in Eq. (36) involve scalar LQs, while the others involve vector types. The LQs in the first and third rows have fermion number $F=3 B+L$ $=0$, while the others have $F=-2$. The indices refer to their isospin representation.

A recent global analysis of scalar LQ constraints from EW data is given in Ref. [84]. Here, we extend this analysis to include vector LQ interactions. We also update it by including the new $Q_{W}(\mathrm{Cs})$ in Eq. (3), hadronic production cross sections at LEP 2 up to $207 \mathrm{GeV}$ [70], and the analysis of nuclear $\beta$ decay given in Ref. [85]. We only consider one LQ

TABLE I. Possible impact of LQ interactions on $Q_{W}(p)$. The left-hand side shows scalar and the righthand side vector LQ species. The columns denote consistency which gives the fractions of the distribution of operator coefficients having the same sign as implied by the LQ model. The final columns give the fractional shifts in $Q_{W}(p)$ allowed by the data. In more statistical terms, consistency is the result of a hypothesis test, while the shifts in $Q_{W}(p)$ reflect parameter estimations that are irrespective of the outcome of the hypothesis test.

\begin{tabular}{lccccc}
\hline \hline LQ & Consistency & $\Delta Q_{W}(p) / Q_{W}(p)$ & LQ & Consistency & $\Delta Q_{W}(p) / Q_{W}(p)$ \\
\hline$S_{1}^{L}$ & 0.57 & $9 \%$ & $U_{1 \mu}^{L}$ & 0.26 & $-8 \%$ \\
$S_{1}^{R}$ & 0.01 & $-6 \%$ & $U_{1 \mu}^{R}$ & 0.56 & $6 \%$ \\
$\widetilde{S}_{1}^{R}$ & 0.44 & $-6 \%$ & $\widetilde{U}_{1 \mu}^{R}$ & 0.99 & $25 \%$ \\
$S_{3}$ & 0.76 & $10 \%$ & $U_{3 \mu}$ & 0.31 & $-4 \%$ \\
$R_{2}^{L}$ & 0.44 & $-13 \%$ & $V_{2 \mu}^{L}$ & 0.87 & $9 \%$ \\
$R_{2}^{R}$ & 0.89 & $15 \%$ & $V_{2 \mu}^{R}$ & 0.11 & $-7 \%$ \\
$\widetilde{R}_{2}^{L}$ & 0.13 & $-4 \%$ & $\widetilde{V}_{2 \mu}^{L}$ & 0.56 & $14 \%$ \\
\hline \hline
\end{tabular}


species at a time. We fit the data and determine the consistency (shown in Table I) of the result with the sign predicted by a given LQ model. The latter is the probability, conditional on the data, that the coefficient has the same sign as implied by the model. For example, the data favor the presence of $\widetilde{U}_{1 \mu}^{R}$, while $S_{1}^{R}$ is virtually excluded. Assuming a given LQ model, we then determine the 95\% C.L. upper limit on $Q_{W}(p)$. Note that this involves a renormalization to the physical parameter space of the model. We observe that the LQ model most favored by the data is $\widetilde{U}_{1 \mu}^{R}$ for which shifts in $Q_{W}(p)$ as large as $25 \%$ are allowed. Since the impact of LQs on $Q_{W}(e)$ is loop suppressed, one would not expect it to deviate significantly from the SM prediction. Thus, if one observes a large effect in $Q_{W}(p), Q_{W}(e)$ would serve as a diagnostic tool to distinguish LQ effects from SUSY.

\section{CONCLUSIONS}

Precise measurements of relatively low energy EW observables continue to play an important part in the search for physics beyond the SM. When taken in the proper context, such studies can provide unique clues about the nature of the EW symmetry breaking, grand unification, etc. We have shown that the weak charge of the proton constitutes a theoretically clean probe of new physics. Presently uncalculable, non-perturbative QCD effects are either sufficiently small or can be constrained by the current program of parity violation measurements so as to render $Q_{W}(p)$ free from potentially worrisome nucleon structure uncertainties. Within the SM, a $4 \%$ determination of $Q_{W}(p)$-as planned at JLab-would yield a $9 \sigma$ determination of the running of the weak mixing angle. Looking beyond the SM, a measurement at this level would provide an effective diagnostic tool for new physics, particularly when considered in tandem with complementary precision low energy studies, such as the SLAC PV Møller scattering experiment, cesium APV, $a_{\mu}, \beta$ decay, and others. Should future experimental developments make an even more precise $Q_{W}(p)$ measurement possible, the physics impact would be correspondingly more powerful. Given its theoretical interpretability, pursuing such experimental developments appear to be well worth the effort.

\section{ACKNOWLEDGMENTS}

We thank K. Cheung, A. Derevianko, V. Flambaum, P. Herczeg, P. Langacker, W. Marciano, S. Su, and O. P. Sushkov for helpful discussions. This work was supported by the U.S. Department of Energy contracts DE-FG0200ER41146, DE-FG03-02ER41215, DE-FG0300ER4112, by NSF contract PHY00-71856, by CONACYT (México) contract 42026-F, and by DGAPA-UNAM contract PAPIIT IN112902.
[1] P. Langacker, Precision Tests of the Standard Electroweak Model, Advanced Series on Directions in High Energy Physics Vol. 14 (World Scientific, Singapore, 1995).

[2] M.J. Ramsey-Musolf, Phys. Rev. C 60, 015501 (1999).

[3] M.J. Ramsey-Musolf, Phys. Rev. D 62, 056009 (2000).

[4] J. Erler and P. Langacker, in [5], p. 98.

[5] Particle Data Group, K. Hagiwara et al., Phys. Rev. D 66, 010001 (2002).

[6] Muon $g-2$ Collaboration, G.W. Bennett et al., Phys. Rev. Lett. 89, 101804 (2002).

[7] NuTeV Collaboration, G.P. Zeller et al., Phys. Rev. Lett. 88, 091802 (2002).

[8] M. Knecht and A. Nyffeler, Phys. Rev. D 65, 073034 (2002).

[9] M. Ramsey-Musolf and M.B. Wise, Phys. Rev. Lett. 89, 041601 (2002).

[10] M. Davier, S. Eidelman, A. Höcker, and Z. Zhang, Eur. Phys. J. C 27, 497 (2003).

[11] K. Hagiwara, A.D. Martin, D. Nomura, and T. Teubner, Phys. Lett. B 557, 69 (2003).

[12] A. Bodek et al., Phys. Rev. Lett. 83, 2892 (1999).

[13] S. Davidson et al., J. High Energy Phys. 02, 037 (2002).

[14] QWEAK Collaboration, R. Carlini et al., "The Q(Weak) Experiment: A Search for Physics at the TeV Scale Via a Measurement of the Proton's Weak Charge," JLAB Report No. E-02-020.

[15] SLAC-E-122 Collaboration, C.Y. Prescott et al., Phys. Lett. 84B, 524 (1979).

[16] CERN-NA-004 Collaboration, A. Argento et al., Phys. Lett. 120B, 245 (1983).
[17] W. Heil et al., Nucl. Phys. B327, 1 (1989).

[18] P.A. Souder et al., Phys. Rev. Lett. 65, 694 (1990).

[19] SAMPLE Collaboration, R. Hasty et al., Science 290, 2117 (2000).

[20] HAPPEX Collaboration, K.A. Aniol et al., Phys. Lett. B 509, 211 (2001).

[21] N.H. Edwards et al., Phys. Rev. Lett. 74, 2654 (1995).

[22] P.A. Vetter et al., Phys. Rev. Lett. 74, 2658 (1995).

[23] C.S. Wood et al., Science 275, 1759 (1997).

[24] SLAC-E-158 Collaboration, E.W. Hughes et al., "A Precision Measurement of the Weak Mixing Angle in Mbller Scattering," SLAC Report No. E-158.

[25] A. Derevianko, Phys. Rev. Lett. 85, 1618 (2000).

[26] V.A. Dzuba, C. Harabati, W.R. Johnson, and M.S. Safronova, Phys. Rev. A 63, 044103 (2001).

[27] M.G. Kozlov, S.G. Porsev, and I.I. Tupitsyn, Phys. Rev. Lett. 86, 3260 (2001).

[28] A.I. Milstein and O.P. Sushkov, Phys. Rev. A 66, 022108 (2002).

[29] W.R. Johnson, I. Bednyakov, and G. Soff, Phys. Rev. Lett. 87, 233001 (2001).

[30] V.A. Dzuba, V.V. Flambaum, and J.S. Ginges, Phys. Rev. D 66, 076013 (2002).

[31] M.Y. Kuchiev and V.V. Flambaum, Phys. Rev. Lett. 89, 283002 (2002).

[32] A.I. Milstein, O.P. Sushkov, and I.S. Terekhov, Phys. Rev. Lett. 89, 283003 (2002).

[33] M.J. Musolf et al., Phys. Rep. 239, 1 (1994). 
[34] A4 Collaboration, D. von Harrach et al. (unpublished).

[35] GØ Collaboration, D.H. Beck et al., "Parity Violation in ep and eD Scattering: The G $\emptyset$ Experiment," JLAB Report No. E-00-006.

[36] M.A. Bouchiat and J. Guéna, J. Phys. (France) 49, 2037 (1988).

[37] S.C. Bennett and C.E. Wieman, Phys. Rev. Lett. 82, 2484 (1999).

[38] S.J. Pollock and M.C. Welliver, Phys. Lett. B 464, 177 (1999).

[39] B.Q. Chen and P. Vogel, Phys. Rev. C 48, 1392 (1993).

[40] D. Vretenar, G.A. Lalazissis, and P. Ring, Phys. Rev. C 62, 045502 (2000).

[41] P.K. Panda and B.P. Das, Phys. Rev. C 62, 065501 (2000).

[42] M.E. Peskin and T. Takeuchi, Phys. Rev. Lett. 65, 964 (1990).

[43] M. Veltman, Nucl. Phys. B123, 89 (1977).

[44] A. Djouadi and C. Verzegnassi, Phys. Lett. B 195, 265 (1987).

[45] A. Djouadi, Nuovo Cimento Soc. Ital. Fis., A 100, 357 (1988).

[46] L. Avdeev, J. Fleischer, S. Mikhailov, and O. Tarasov, Phys. Lett. B 336, 560 (1994).

[47] K.G. Chetyrkin, J.H. Kühn, and M. Steinhauser, Phys. Lett. B 351, 331 (1995).

[48] R. Barbieri et al., Phys. Lett. B 288, 95 (1992); Nucl. Phys. B409, 105 (1993).

[49] J. Fleischer, O.V. Tarasov, and F. Jegerlehner, Phys. Lett. B 319, 249 (1993).

[50] G. Degrassi, P. Gambino, and A. Vicini, Phys. Lett. B 383, 219 (1996).

[51] W.J. Marciano and A. Sirlin, Phys. Rev. D 27, 552 (1983); 29, 75 (1984)

[52] M.J. Musolf and B.R. Holstein, Phys. Rev. D 43, 2956 (1991).

[53] M.J. Musolf and B.R. Holstein, Phys. Lett. B 242, 461 (1990).

[54] A. Sirlin, Rev. Mod. Phys. 50, 573 (1978).

[55] S.L. Adler and W.K. Tung, Phys. Rev. Lett. 22, 978 (1969).

[56] M.A.B. Bég, Phys. Rev. D 11, 1165 (1975).

[57] W.J. Marciano, Precision Tests of the Standard Electroweak Model, Advanced Series on Directions in High Energy Physics Vol. 14 [1], p. 170.

[58] J. Erler, "Determinations of $\alpha\left(M_{Z}\right)$ : Comparison and Prospects," hep-ph/0111005.

[59] J. Erler, Phys. Rev. D 59, 054008 (1999).

[60] Snowmass Working Group on Precision Electroweak Measurements, U. Baur et al., "Present and Future Electroweak Preci- sion Measurements and the Indirect Determination of the Mass of the Higgs Boson," hep-ph/0202001.

[61] J. Erler and M. Luo, Phys. Lett. B 558, 125 (2003).

[62] M. Cvetič and P. Langacker, ' $\mathrm{Z}$ ' Physics and Supersymmetry," hep-ph/9707451.

[63] P. Langacker, “ $Z$ ' Physics from Strings,” hep-ph/9805486.

[64] M. Cvetič and P. Langacker, Phys. Rev. D 54, 3570 (1996).

[65] J.E. Kim and H.P. Nilles, Phys. Lett. 138B, 150 (1984).

[66] J. Erler, Nucl. Phys. B586, 73 (2000).

[67] CDF Collaboration, F. Abe et al., Phys. Rev. Lett. 79, 2192 (1997).

[68] CDF Collaboration, T. Affolder et al., Phys. Rev. Lett. 87, 131802 (2001).

[69] J. Erler, P. Langacker, and T. Li, Phys. Rev. D 66, 015002 (2002).

[70] ALEPH, DELPHI, L3, and OPAL Collaborations, LEP Electroweak Working Group, and SLD Heavy Flavour Working Group, D. Abbaneo et al., "A Combination of Preliminary Electroweak Measurements and Constraints on the Standard Model," hep-ex/0212036.

[71] J. Erler and P. Langacker, Phys. Rev. Lett. 84, 212 (2000).

[72] P. Langacker and M. Plümacher, Phys. Rev. D 62, 013006 (2000).

[73] P. Langacker and M. Luo, Phys. Rev. D 45, 278 (1992).

[74] S. Dimopoulos and D.W. Sutter, Nucl. Phys. B452, 496 (1995).

[75] H.E. Haber, Nucl. Phys. B (Proc. Suppl.) 62A, 469 (1998).

[76] A. Kurylov and M.J. Ramsey-Musolf, Phys. Rev. Lett. 88, 071804 (2002).

[77] A. Kurylov, M.J. Ramsey-Musolf, and S. Su, "Parity-violating Electron Scattering as a Probe of Supersymmetry," hep-ph/0205183.

[78] A. Kurylov, M.J. Ramsey-Musolf, and S. Su, "Supersymmetric Effects in Deep Inelastic Neutrino Nucleus Scattering," hep-ph/0301208.

[79] L.J. Hall and M. Suzuki, Nucl. Phys. B231, 419 (1984).

[80] B.C. Allanach, A. Dedes, and H.K. Dreiner, Phys. Rev. D 60, 075014 (1999).

[81] J.C. Pati and A. Salam, Phys. Rev. Lett. 32, 1083 (1974).

[82] G. Senjanovic and A. Sokorac, Z. Phys. C 20, 255 (1983).

[83] W. Buchmüller, R. Rückl, and D. Wyler, Phys. Lett. B 191, 442 (1987).

[84] K.M. Cheung, Phys. Lett. B 517, 167 (2001).

[85] I.S. Towner and J.C. Hardy, Phys. Rev. C 66, 035501 (2002). 